\title{
Die Entstehung des Begriffs der Zivilisationskrankheiten
}

Von Carl Haffter

Das Wort Zivilisationskrankheit tritt erst im 20. Jahrhundert auf; vorher sprach man von Culturkrankheiten (Arndt ${ }^{3}$ 1885, Stekel ${ }^{27}$ 1907) oder von Culturschäden (Gelpke $\left.{ }^{13} 1905\right)$; aber der Begriff ist fast 200 Jahre älter. Wir verstehen darunter die Vorstellung, daß Veränderungen der Lebensverhältnisse neue Krankheitszustände hervorrufen oder zur Vermehrung schon bekannter Krankheiten führen. Ich beschränke mich dabei auf jene Störungen, die von Delius und Fahrenberg ${ }^{8}$ als «psychovegetative Syndrome» umschrieben werden und auch von Dittmar ${ }^{9}$ unter dem Titel «Zivilisation und Nervenkrankheiten» behandelt werden. Es ist jene Gruppe von Zustandsbildern, die nach den Arbeiten von Frau E. FischerHomberger ${ }^{12}$ seit Anfang des 18. Jahrhunderts unter wechselnden Namen beschrieben, aber immer wieder den Einflüssen der modernen Zivilisation zugeschrieben wurden. Die Reihe führt von der Hypochondrie über Spinalirritation bzw. Cerebralirritation oder Nervosismus zur Neurasthenie, dann zur Neurose und in der Gegenwart zur vegetativen Dystonie, zu den Streßkrankheiten und den psychosomatischen Leiden.

Die Bezeichnung des allgemeinen Zustandes eines Volkes bzw. einer Epoche als «Kultur» entwickelte sich im 18. Jahrhundert (Niedermann ${ }^{22}$ ). In der gleichen Zeit entstand der Begriff des «Fortschritts». Die neuzeitliche Idee eines stetigen Fortschreitens der Menschheitsgeschichte steht im Gegensatz zur antiken Vorstellung der aufeinanderfolgenden Lebensalter der Menschheit, aber auch zur christlichen Erwartung des Weltendes mit dem Wiedererscheinen Christi. Die Aufklärung brachte anstelle des stets zu erwartenden Endes der Weltzeit die säkularisierte Vorstellung einer Bewegung der Weltgeschichte auf eine offene Zukunft hin (Koselleck ${ }^{16}$, Löwith ${ }^{17}$ ).

Das Wort war eine Übersetzung des französischen progrès, das zuerst mit Bezug auf einzelne Aspekte der Kultur verwendet wurde. Rousseau ${ }^{24}$ spricht vom "progrès des sciences et des arts». Als Verallgemeinerung wurde zuerst der Plural «les progrès» verwendet, auch deutsch als «die Progresse». Schließlich setzte sich aber der Kollektivsingular Fortschritt durch, der im 19. Jahrhundert vulgarisiert und zum politischen Schlagwort werden sollte. Es kann jetzt vom Fortschrittsglauben und von Fortschrittskritik schlechthin gesprochen werden. 
Eine frühe Form von Fortschrittskritik, von einem Zweifel an den Segnungen des Fortschritts, ist die Vorstellung von den Zivilisationskrankheiten, wie sie im England des frühen 18. Jahrhunderts auftaucht. England galt damals als das typische Land eines Leidens, das unter dem Namen Hypochondrie (auch abgekürzt Hypo oder Hyp) lief, aber vor allem auch als Spleen bezeichnet wurde ${ }^{12}$. Beide Namen gingen auf die antike Medizin zurück, welche die Krankheit Melancholie nach der antiken Viersäftelehre mit der schwarzen Galle in Zusammenhang brachte, die aus der Milz (lat. splen) stammen sollte. Die Hypochondrie war ein Abkömmling der Melancholie, zuerst eine Unterform, dann eine selbständige Krankheit, wobei man heute sagen kann, daß der Begriff Melancholie damals mehr psychotische Zustände, die Begriffe Hypochondrie und Spleen mehr funktionellpsychogene bezeichneten. Jedoch wurden in der Umgangssprache jener Zeit auch alle drei Ausdrücke nebeneinander gleichsinnig verwendet.

Zur Symptomatik der Hypochondrie gehörten Beschwerden im Hypochondrium, d.h. Störungen der Magen- und Darmfunktion. Die Ableitung von einer «Milzsucht» war jedoch seit Sydenham ${ }^{29}$ aufgegeben worden. Er hatte 1681 eine Begriffsklärung zustande gebracht, insofern als er zeigte, daß die gleiche Gruppe von Symptomen, die man bei Männern als hypochondrische zu bezeichnen pflegte, bei Frauen Hysterie oder «Vapours» (nach dem franz. vapeurs) genannt würden. Der Spleen habe ebensowenig mit der Milz wie die Hysterie mit dem Uterus zu tun. Beides sei die gleiche Nervenkrankheit. Er fand dieses Leiden bei einem Sechstel seiner Patienten; das mache die Hälfte aller chronischen Krankheiten aus. Er fand es besonders häufig bei Frauen, außer bei solchen, die hart arbeiteten. Von den Männern seien besonders jene befallen, die eine sitzende Lebensweise hätten und intensiv studierten. Diese Zuordnung ging auf eine ehrwürdige Tradition zurück. Das in England so populäre Buch The Anatomy of Melancholy von Burton ${ }^{6}$ (1621) nennt als wichtige Ursache «Love of Learning» und «Overmuch Study» und widmet den «Miseries of Scholars» 30 Seiten mit häufigen Zitaten aus Ficinus und andern Renaissanceautoren.

Gegen Ende des 17. Jahrhunderts finden sich nun Hinweise darauf, daß der Spleen in England besonders häufig sei. Temple ${ }^{30}$ schreibt 1690: «Our country must be confess'd to be what a great foreign Physician called it, the Region of the Spleen. » 1711/12 schreibt Addison ${ }^{2}$ wiederholt im Spectator darüber und bringt schließlich das eindrucksvolle Bild: "Melancholy is a kind of Demon that haunts our Island, and often conveys her self to us in an Easterly Wind». In gleichem Sinne spricht er auch von Gloominess und von Spleen. Er kennt als Ursache 
ebenfalls die sitzende Lebensweise und das Studium und empfiehlt dagegen Reiten und gymnastische Übungen. Sein Hinweis auf den Ostwind entspricht einer weitverbreiteten Erklärung der depressiven Zustände der Engländer, die ja auch als besonders suizidgefährdet galten. In vielen Reisebeschreibungen von ausländischen Besuchern wird auf das deprimierende Klima hingewiesen, und die Engländer selber klagen es an, das feuchte, neblige Wetter, speziell aber den Ostwind (Moore $\left.{ }^{18}\right)$.

Die Ärzte übernahmen die populären Ansichten von der «Nationalkrankheit» Hypochondrie. Sie nannten sie in ihren Schriften Morbus anglicus (Harvey $1666^{15}$ ) oder The English Malady (Cheyne 1733) ${ }^{7}$. Die Auffassung als Zivilisationskrankheit wurde von zwei Männern, Swift und Cheyne, vertreten, die eng miteinander befreundet waren, so daß die Priorität keine Rolle spielen dürfte. Zudem hatte Cheyne schon 1724 in seinem «Essay on Health and Long Life» die falsche Ernährung und den Alkoholgenuß der Engländer angekreidet, für manche schwere Leiden vegetarische und alkoholfreie Diät empfohlen, und damit einen Sturm der Entrüstung hervorgerufen. Wir beginnen also nicht mit ihm und seinem Hauptwerk, sondern mit der Gesellschaftskritik von Swift, in seinen Gullivers Reisen $(1726)^{28}$. Im vierten Teil, der Reise ins Land der Houyhnhnms, wird der Reisende von der meuternden Mannschaft auf einer Insel ausgesetzt und lernt einen idealen Pferdestaat kennen, wo die Rollen von Pferden und Menschen vertauscht sind. Er lernt während seines dreijährigen Aufenthaltes die Pferdesprache verstehen und sprechen und erzählt nun seinem Herrn, einem edlen Pferd, von den Zuständen in seiner Heimat England. Die gesund lebenden Pferde kennen keine Krankheiten, und Gulliver hat Mühe, dem Pferd den Begriff der Krankheit begreiflich zu machen.

«Daß aber die Natur, die alle Dinge zur Vollkommenheit bringt, Schmerzen in unserm Körper keimen lassen sollte, hielt er für unmöglich, und er wünschte die Ursache eines so unerklärlichen Übels zu wissen. Ich sagte ihm, wir nährten uns von tausend Dingen, die einander zuwiderwirkten; wir äßen, wenn wir nicht hungrig wären, und tränken, ohne vom Durst gereizt zu sein; wir säßen ganze Nächte lang beisammen und schlürften starkwirkende Getränke, ohne einen Bissen dazu zu essen, und das mache uns zur Trägheit geneigt, entzünde uns den Leib und beschleunige oder verzögere die Verdauung. Feile weibliche Yahoos (Menschen) zögen sich eine bestimmte Krankheit zu, die in den Gebeinen derer, die sich ihren Umarmungen hingäben, Fäulnis erzeuge; diese und andere Krankheiten pflanzten sich vom Vater auf den Sohn fort, so daß zahlreiche schon mit vielfältigen Krankheiten behaftet zur Welt kämen; ich würde 
zu keinem Ende kommen, wollte ich ihm ein Verzeichnis aller Leiden geben, die den menschlichen Leib heimsuchten; denn es waren ihrer nicht weniger als fünf- oder sechshundert, die sich über alle Glieder und Gelenke erstreckten; kurz, jeder innere und äußere Körperteil habe bei uns die ihm eigentümlichen Krankheiten ...

Abgesehen aber von den wirklichen Krankheiten leiden wir unter vielen, die nur eingebildet sind, und für die auch die Ärzte eingebildete Heilmethoden erfunden haben; die besitzen ihre verschiedenen Namen und ebenso auch die Arzneien; und mit diesen Krankheiten sind unsere weiblichen Yahoos stets behaftet.»

In einem andern Gespräch erwähnt das Herrenpferd folgende Beobachtung:

«Bisweilen komme einen Yahoo die Grille an, daß er sich in einen Winkel zurückziehe und sich niederlege und heule und stöhne und alle fortjage, die sich ihm nahten, und zwar, obwohl er jung und fett sei und weder an Futter noch an Wasser Mangel leide; und auch seine Diener könnten sich nicht vorstellen, was ihn anfechten möge. Das einzige Heilmittel, das sie gefunden hätten, bestehe darin, ihn harte Arbeit verrichten zu lassen, denn die bringe ihn unfehlbar zur Besinnung. Ich schwieg aus Parteilichkeit für meine eigene Gattung; doch konnte ich hier deutlich die wahren Keime des Spleens erkennen, der nur die Müßigen, Üppigen und Reichen packt; und wenn man sie derselben Kur unterwürfe, so würde ich mich gern für die Heilung verbürgen.»

Vom exzessiven Wohlstand seiner Heimat gibt Gulliver folgende Schilderung:

«Deshalb wünschte er, ich möchte ihm sagen, was für kostspielige Speisen das seien ... Ich erklärte ihm die verschiedenen Arten, sie zuzubereiten; sie würden nur dadurch ermöglicht, daß man zur See in alle Teile der Welt Schiffe entsende, sowohl um Getränke wie auch Saucen und unzählige andere Waren zu holen. Ich versicherte ihm, daß man mindestens erst dreimal um die Erde fahren müßte, bevor eins unserer vornehmen Yahooweibchen ihr Frühstück habe, oder eine Tasse, daraus zu trinken ...

Um aber die Üppigkeit und Maßlosigkeit der Männchen und die Eitelkeit der Weibchen zu befriedigen, schickten wir den größern Teil unserer notwendigen Dinge in andere Länder, von denen wir dafür die Grundstoffe von Krankheiten, Narrheiten und Lastern erhielten, die wir unter uns verbrauchten ...

Wenn ich zu Hause sei und angezogen, wie es sich gehöre, so trüge ich auf meinem Leibe die Arbeit von hundert Handwerkern; der Bau und die Einrichtung meines Hauses beschäftige noch einmal soviel und der Putz meines Weibes die fünffache Zahl.» 
Cheyne bringt gleich im Vorwort seines Buches The English Malady (1733) eine Zusammenfassung seiner These ${ }^{7}$ :

«Der Titel, den ich für diese Abhandlung gewählt habe, ist ein Vorwurf, der von Fremden und unsern Nachbarn auf dem Kontinent ganz allgemein gegen unser Land erhoben wird. Es ist nämlich der Spottname «Englische Krankheit» aufgekommen für jene Störungen, die man Nervous Distempers, Spleen, Vapours und Lowness of Spirits nennt. Ich wünschte, es gäbe nicht so gute Gründe für diese Bezeichnung. Die Feuchtigkeit unserer Luft, die Veränderlichkeit unseres Wetters (wegen der Lage unserer Insel mitten im Meer), die Üppigkeit und Fruchtbarkeit unseres Bodens, unsere reichhaltige und schwere Nahrung, der Wohlstand und Überfluß, in dem wir leben (bedingt durch unseren Welthandel), der Mangel an körperlicher Bewegung und die sitzenden Beschäftigungen unserer höheren Bevölkerungsschichten (in welchen das Übel am meisten verbreitet ist) und die Tendenz, in großen, dichtbevölkerten und deshalb ungesunden Städten zu leben, - all dies hat zu einer Gruppe von Störungen mit schrecklichen Symptomen geführt, die unseren Vorfahren kaum bekannt waren und die in solcher Häufigkeit in keiner anderen Nation vorkommen. Diese Störungen machen nahezu einen Drittel aller Beschwerden aus, an denen die vornehmen Leute in England leiden.»

Die Zivilisationsschäden, die Cheyne anprangert, lassen sich auf drei Punkte zurückführen: falsche Ernährung, Mangel an körperlicher Bewegung, ungesunde Stadtluft (in Verbindung mit dem bekanntlich schlechten Klima). Die Zivilisationskritik Cheynes ist bitter:

«Mit zunehmendem Wohlstand und mit der Ausdehnung unserer Schiffahrt haben wir alle Erdteile ausgeraubt, nur um bei uns Vorräte aufzustapeln, die dem Luxus, der Verschwendung und Ausschweifung dienen müssen. Die Tische der Reichen und Großen (und aller, die es sich leisten können) sind beladen mit einem Angebot der verschiedenartigsten und raffiniertesten Genüsse, die selbst den gierigsten Appetit reizen und befriedigen können. Jeder sucht den andern in dieser Verschwendung zu übertreffen. Man zerbricht sich den Kopf, um die Speisen möglichst schmackhaft und raffiniert zuzubereiten.»

Anstatt die Tiere natürlich aufwachsen zu lassen, werden diese eingesperrt, gestopft und allen möglichen Torturen unterworfen, um ihr Fleisch schmackhafter zu machen. Sie bekommen eine unnatürliche, scharf gewürzte Nahrung (they are physick'd); dadurch werden ihr Fleisch und ihre Säfte vergiftet, und es werden bei ihnen dadurch selbst schon jene nervösen Störungen erzeugt, an denen jene leiden, welche sie verspeisen. Auch die gebrannten Wässer dienen als Vehikel 
für die ungesunde Nahrung; sie müssen dem vollgestopften Magen helfen, die Speisen zu verdauen, und dazu anreizen, mit der Völlerei weiterzufahren.

Diese Lebensweise führt dazu, daß man zu körperlicher Bewegung unfähig wird. Man vermeidet möglichst jede Anstrengung und Bewegung. Die Kutschen werden zu größerer Bequemlichkeit mit Federn versehen; man hält sich Pferde zum Fahren und Reiten; man stellt Kutscher an. (Der Popularisator Bilguer wird 1767 diese Art der zunehmenden «Motorisierung» durch die Angabe belegen, es treffe in England bereits auf jedes Haus ein Pferd ${ }^{4}$.) Ist es ein Wunder, schließt Cheyne, daß die Krankheiten, die von Trägheit und Überfütterung herrühren, im gleichen Maße zugenommen haben, wie die Fortschritte unseres Wohlstandes?

Die alte Theorie der Prädisposition der «Scholars» zur Melancholie bzw. Hypochondrie wird von Cheyne sehr geschickt in seine Fortschrittskritik einbezogen, und hier verläßt er den anklagenden Ton des eifernden Moralisten : die ungesunde Lebensweise ist eine Kehrseite des positiven kulturellen Fortschritts:

"Now since this present Age has made Efforts to go beyond former Times, in all the Arts of Ingenuity, Invention, Study, Learning, and all the contemplative and sedentary Professions, (I speak only here of our Nation, our own Times, and of the better Sort, whose chief Employments and Studies these are) the Organs of these Faculties being thereby worn and spoil'd, must affect and deaden the whole System, and lay a Foundation for the Diseases of Lowness and Weakness. Add to this, that those who are likeliest to excel and apply in this manner, are most capable, and most in hazard of following that Way of Life which I have mentioned, as the likeliest to produce these diseases."

Zum Schluß schildert Cheyne die Luftverschmutzung als zusätzliche Ursache der englischen Krankheit. London ist die größte und volkreichste Stadt der Welt. Die unzähligen Feuerstätten, schweflig und bituminös, der riesige Verbrauch von Talg und Öl in Kerzen und Lampen, die Wolken von stinkendem Atem und Schweiß, die Fäulnis verendeter Tiere, die Friedhöfe mit vermodernden Leichen, die Abwässer, Schlachthäuser, Ställe, Miststöcke - dadurch wird die Luft im Umkreis von 20 Meilen verpestet. Dies muß mit der Zeit die gesundeste Konstitution aller Lebewesen zerstören. Darum kommen die erwähnten Störungen besonders häufig in solchen Städten vor. Selten kommt eine Heilung zustande, wenn der Kranke nicht aufs Land gebracht und dadurch von den giftigen Dämpfen gereinigt wird.

Frau Fischer-Homberger hat in ihrer Monographie über die Hypochondrie dargestellt, wie die Hypochondrie zur Modekrankheit wurde und wie es über sie 
eine Unzahl von populären Aufklärungsschriften von Ärzten und andern Autoren gab. Wir lesen etwa bei Bilguer (1767), daß sie nicht nur eine englische Krankheit sei, sondern in allen zivilisierten Ländern vorkomme; auch sei sie nicht mehr auf die obern Stände und die Gelehrten beschränkt, sondern «eine fast allgemeine Krankheit». ${ }^{4}$ Ackermann in seiner (sehr freien) Übersetzung und Ergänzung von Ramazzinis Arbeit über die Krankheiten der Künstler und Handwerker (lat. Urtext 1700, Ackermanns deutscher Text 1780) datiert die Zunahme der Nervenkrankheiten «seit etwa 50 Jahren»., ${ }^{1,23}$ Tissot schreibt $1778 \mathrm{im}$ «Traité des nerfs et de leur maladies», diese hätten seit Beginn des Jahrhunderts unstreitig zugenommen ${ }^{31}$. Bei jedem dieser drei Autoren kommen jedoch jetzt Argumente hinzu, die auf Rousseau zurückgehen, der inzwischen seine beiden Discours $(1750,1754)$ veröffentlich hatte ${ }^{24}$.

\section{Rousseaus Zivilisationskritik}

Der Zweifel, den Rousseau gegenüber dem Fortschritt äußerte, scheint unabhängig von Swift und Cheyne entstanden zu sein. Der Mann, der für ihn und für Haller von bestimmendem Einfluß war, ist Beat Ludwig von Muralt. Dieser hatte zwischen 1694 und 1698 seine Reisebriefe geschrieben, die indessen erst 1725 publiziert wurden: «Lettres sur les Anglois, lettres sur les François, lettres sur les voiages ${ }^{20}$ Von Muralt kritisierte die damalige Mode der Auslandreisen, auf welche man die jungen Männer schickte, um sie zu bilden. Nach seiner Auffassung brachten sie schlechte Vorbilder und ungute Einflüsse in ihre schlichte Heimat zurück. Davor wollten seine Schriften sein Vaterland bewahren. «Heureuse Nation, si elle revenoit à soi, et si elle savoit jouir de ses avantages. La Simplicité et la Droiture ont été son partage.» Dasselbe Pathos finden wir in Hallers Dichtung «Die Alpen». ${ }^{14}$ Nicht einmal das Erlernen fremder Sprachen genügt von Muralt als Begründung für Auslandsaufenthalte. Man brauche überhaupt nicht soviel Bücher zu lesen; man solle lieber «le grand livre du Monde» kennenlernen, ein Ausdruck, der in Rousseaus «Emile» wiederkehrt.

Neu ist bei Rousseau, daß er als Kontrast zum verderbten Kulturmenschen den Naturmenschen und das Tier hinstellt. Er nimmt damit vor allem Themen von Montaigne ${ }^{19}$ auf, der in seinen Essais einerseits antike Autoren wie Plutarch, anderseits Reiseberichte aus der Neuen Welt zitierte. Es geht Rousseau hauptsächlich um die sittlichen Unterschiede, um die Vorstellung des «noble savage» (Dryden 1670) ${ }^{10}$, die weiterwirkte bis zum Gedicht von Seume (1793), das immer

noch zitiert wird: «Seht, wir Wilden sind doch beß're Menschen. » ${ }^{25,11}$ En passant hatte Montaigne schon referiert, daß Wilde eine bessere Gesundheit hätten als 
zivilisierte Menschen. Den Vergleich mit der Gesundheit der Tiere und der Naturmenschen schöpfte Rousseau aus der «Histoire naturelle» von Buffon ${ }^{5}$, hier wie dort in tendenziöser Verallgemeinerung, die jedoch gerade die nachhaltige Wirkung seiner Schriften ausmachte (Starobinski ${ }^{26}$ ).

Medizinische Autoren zitiert Rousseau kaum. Erst in Anmerkungen zu einer späteren Auflage kommt er auf die Berufskrankheiten in besonders ungesunden Handwerken zu sprechen, schöpft also direkt oder indirekt aus Ramazzini ${ }^{23}$. Man kann gerade an diesem Werk verfolgen, wie sich das Thema im Verlauf dieses Jahrhunderts wandelte. Ramazzinis berühmtes Buch, das als Begründung einer Arbeitsmedizin gilt, ist ohne jede kulturkritische Tendenz geschrieben. Sein deutscher Bearbeiter Ackermann jedoch schreibt 1780 ganz im Sinne von Tissot und Rousseau:

«Mehrmals ist es schon gesagt, und aus der Geschichte durch unwiderlegliche Beweise erhärtet worden, daß sich mit den vermehrten Bedürfnissen des Menschengeschlechts auch die Krankheiten desselben vermehrt haben, und daß der Mensch, je weiter er sich von dem einfachen und heilsamen Pfad der Natur entfernte, auch desto mehreren, größeren und verderblicheren Krankheiten ausgesetzt worden sey. Deswegen trifft auch ein aufmerksamer Reisender in Ländern, wo sich die Menschen durch emporgestiegene Kultur noch nicht veredelt haben, ... wenig Krankheiten, weniger Ärzte und ebenso wenige und einfache Mittel an. ${ }^{23}$

Wir können dieses Kapitel abschließen mit Tissot, in dessen Werk die beiden Strömungen, diejenige von Cheyne/Swift und die von Rousseau, zusammenfließen. Er hat nicht nur auf die Ärzte seiner Zeit, wie z. B. Zimmermann, einen starken Einfluß ausgeübt, sondern auch auf die Laien, die er durch seine Aufklärungsschriften, besonders den Avis au peuple sur la santé, ansprach ${ }^{31}$.

Zugleich zeichnet sich bei ihm ab, wie der Ausdruck Hypochondrie seine zentrale und umfassende Bedeutung verliert. Er spricht ganz allgemein von Nervenkrankheiten, erwähnt darunter auch die Hypochondrie. Unter den Ursachen der zunehmenden Nervenleiden gibt er wohl die drei von Cheyne aufgezählten an; er fügt aber viele andere Zivilisationsnoxen hinzu. Darunter finden wir u.a. den vermehrten Genuß heißer Getränke, speziell der neuaufgekommenen Genußmittel Tee und Kaffee. Schädlich für die Nerven sei auch die spektakuläre Vermehrung der Romanliteratur. Jung und alt, besonders die Frauen, läsen unersättlich Romane bis spät in die Nacht.

«Une fille qui, à l'âge de dix ans, lit quand elle devrait courir, est à vingt une femme à vapeurs, \& non pas une bonne nourrice.» 
Wichtig ist für Tissot auch die Vorstellung der zunehmenden Degeneration der Konstitution. Diese kommt vor allem durch die venerischen Leiden zustande. Nicht weniger schädlich sind die lange fortgesetzten antiluetischen Kuren mit Quecksilber. Bei Tissot kommt schließlich die Warnung vor allen schädlichen sexuellen Exzessen hinzu, die für lange Zeit im Bild der Zivilisationskrankheiten nicht mehr fehlen wird. Er ist mit seiner Anklage gegen die Verderbnis der Sitten und die Abkehr vom natürlichen Leben ein unverkennbarer Rousseau-Anhänger.

\section{Literatur}

1. Joh.Christian Gottlieb Ackermann: s. Ramazzini.

2. Joseph Addison, verschiedene Beiträge im Spectator (1711-1712).

3. Rudolf Arndt, Die Neurasthenie, Wien und Leipzig 1885.

4. Joh. Ulrich Bilguer, Nachrichten an das Publikum in Absicht der Hypochondrie, Kopenhagen 1767.

5. Comte de Buffon, Histoire naturelle, Paris 1749-1804.

6. Robert Burton, The Anatomy of Melancholy, Oxford 1621.

7. George Cheyne, The English Malady, London 1733.

8. Ludwig Delius und Jochen Fahrenberg, Psychovegetative Syndrome, Stuttgart 1966.

9. Friedrich Dittmar, Zivilisation und Nervenkrankheiten, Herford 1969.

10. John Dryden: s. Fairchild.

11. H.N.Fairchild, The noble savage, New York 1928.

12. Esther Fischer-Homberger, Hypochondrie, Bern 1970. - Vergessene Zivilisationskrankheiten, Bern 1979.

13. Ludwig Gelpke, Culturschäden, Basel 1905.

14. Albrecht von Haller, Die Alpen [entstanden 1729], Erstdruck in: Versuch Schweizerischer Gedichten, Bern 1732.

15. Gideon Harvey, Morbus Anglicus, London 1666.

16. Reinhart Koselleck, «Fortschritt» in: Geschichtliche Grundbegriffe, Klett-Verlag, Stuttgart 1972.

17. Karl Löwith, Das Verhängnis des Fortschritts, in: Die Idee des Fortschritts, München 1963.

18. Cecil A. Moore, The English Malady, in: Backgrounds of English literature, Minneapolis 1953.

19. Michel de Montaigne, Essais, Bordeaux 1580.

20. Beat Ludwig von Muralt, Lettres sur les Anglois et les François et sur les voiages, Genf 1725.

21. Murray et al., A new English dictionary, Oxford 1919, Artikel «Spleen».

22. Joseph Niedermann, Kultur. Werden und Wandlungen des Begriffs. In: Biblioteca dell' «Archivum Romanicum», vol. 28, Florenz 1941.

23. Bernardino Ramazzini, De morbis artificium diatriba, Modena 1700. - Deutscher Text, neu bearbeitet und vermehrt, von J.C.G.Ackermann, Stendal 1780/1783. 
24. Jean-Jacques Rousseau, Discours sur les sciences et les arts, 1750. - Discours sur l'inégalité parmi les hommes, 1754. - Emile ou de l'éducation, 1762.

25. Joh. Gottfried Seume, Der Wilde, 1793.

26. Jean Starobinski, J.J.Rousseau. La transparence et l'obstacle. Paris 1971.

27. Wilhelm Stekel, Die Ursachen der Nervosität, Wien 1907.

28. Jonathan Swift, Travels into Several Remote Nations of the World, London 1726. Zitate nach dem deutschen Text, Berlin 1916.

29. Thomas Sydenham, Works: Of the Epidemic Diseases from the Years 1675 to 1680.

30. Sir William Temple: s. Murray: «Spleen».

31. S.A.A.D. Tissot, Traité des nerfs et de leur maladies, Paris 1778. - Avis au peuple sur la santé, Lausanne 1761.

\section{Summary}

At the beginning of the 18 th century, the term "progress" came first into existence. The same time gave rise to the critical examination of the meaning of progress and the notion of "diseases of civilization". According to Swift and Cheyne, the "English Malady" (Hypochondria, Spleen, Nervous Distempers) was due to Englands' Wealth and Universal trade. These illnesses were said to be increasing and affected primarily "the better sort". Soon, they were also recognized among other European countries and among all social classes. Independent of this English trend, Rousseau developed his own general critique of civilization under the influence of v. Muralt and A.v. Haller: he claimed civilization to induce moral decay, but also damages to health. Tissot combined both schools of thought in his writings that exercised a lasting influence on the physicians of his time.

Prof.Dr.med. Carl Haffter

Petersgasse 50

4051 Basel 